\title{
A multivalued agent-based model for the study of noncommunicable diseases
}

\author{
Rabia Aziza ${ }^{\mathrm{a}, *}$, Amel Borgi ${ }^{\mathrm{a}}$, Hayfa Zgaya ${ }^{\mathrm{b}}$, Benjamin Guinhouya ${ }^{\mathrm{b}}$ \\ ${ }^{a}$ Université de Tunis El Manar, Laboratoire LIPAH, LR 11ES14, El Manar 2092, Tunisie \\ ${ }^{b}$ Université de Lille, Laboratoire de Santé Publique, EA 2694: Epidémiologie et Qualité de soins, 42 rue Ambroise Paré 59120, France
}

\begin{abstract}
This paper aims to test and illustrate the utility and extensibility of an existing model, SimNCD (Simulation of NonCommunicable Diseases). It also proposes a way to include questionnaires - widely used in epidemiology - in the individual's reasoning mechanism in order to identify his/her attitude and personal choices.

SimNCD is a formal agent-based model. It helps researchers and health practitioners study and simulate the complex dynamics of noncommunicable diseases. It models individuals that evolve within a social network, and behave while engaging in activities offered by their physical environment. The literature strongly supports the influence of the individual's behavioral choices on their health, particularly, the acquirement and maintainability of noncommunicable diseases. Therefore, we propose to extend SimNCD in order to acquire the agents with a reasoning process that allows them to choose the activities to practice. Thus, we model their attitude via preferences that are modeled based on the available literature and expressed with the linguistic 2-tuple method. Our solution also employs a multi-attribute decision-making method. We specify the proposed solution in the study of childhood obesity and use it to predict children's corpulence variations in different scenarios.
\end{abstract}

Keywords: Agent-based modeling, decision-making, linguistic 2-tuple, noncommunicable diseases, childhood obesity.

\section{Introduction}

Simulation is widely used in public health, more specifically, the study of NonCommunicable Diseases ( $N C D s)$. The risk factors that maintain and lead to NCDs are com- 30 5 plex and dynamic, e.g. social support, physical activity, tobacco use, and neighborhood safety. They stem from different dimensions of a person's life, e.g. social network, physical environment, and psychological level. They can influence each other and may lead to developing other dis- 35 10 eases within the same person, which complicates their understanding and control. Nevertheless, public health researchers and practitioners believe these diseases are preventable if the right factors were targeted [1, 2, 3].

Agent-Based Models ( $A B M s$ ) represent autonomous, in15 dependent agents that evolve within social and physical ${ }^{40}$ environments. This paradigm is known to better suit representing complex and nonlinear dynamics. Therefore, it is often used to model NCDs 4, 5, 6, 7, 8, like the study of the effect of transport infrastructure on walking [9], and the study of the effectiveness of interventions aimed at re- ${ }_{45}$ ducing early childhood obesity [10].

SimNCD (Simulation of NonCommunicable Diseases) 11 is a formal ABM that aims to help researchers and health practitioners study and simulate the dynamics of

\footnotetext{
* Corresponding author

Email address: rabia.aziza@gmail.com (Rabia Aziza)
}

network, and behave while engaging in activities offered by their physical environment. SimNCD allows modeling, among other examples, social interactions, psychological risk factors, behavioral aspects, and environmental factors. However, some specific areas of the study of NCDs could fall beyond the reach of SimNCD, such as the genetic aspect or molecular biology.

In fact, agents in ABMs often rely on human-like reasoning mechanisms that can be based on mental attitudes, e.g. objectives, desires, beliefs, and preferences [12, 13, 14]. Such mechanisms allow them to understand their environment and make decisions that suit their local goals [13, 14.

This paper has three objectives:

- First, we are looking to propose a way to model a reasoning mechanism that describes the individual's attitude when choosing what to do (an activity to practice). For that, we rely on the available literature in epidemiology, as well as the decision-making.

- Secondly, we aim to provide a solution that allows public health practitioners to simulate and predict the influence of such choices on the individual's health.

- Thus, we extend SimNCD to include a mechanism that models such reasoning. This leads us to the third objective which is to test and illustrate the utility and extensibility of SimNCD.

To summarize, we focus on modeling a decision-making process based on the mental attitude of preferences. 
The current paper is structured as follows. In Section 2 , we briefly describe SimNCD. Then, we review the lit-105 55 erature about modeling reasoning mechanisms in NCD ABMs. Section 3 proposes our solution: a preference based decision-making process for the study of NCDs. Then, we focus on the case study of childhood obesity, where we model the indirect effects of children's preferences on the 60 development of their corpulence via their physical activity behaviors. The experimentation about this case study is ${ }^{110}$ described in Section 4. Finally, we conclude in Section 5.

\section{Background}

We begin by describing the formal model SimNCD 11 65 on which our method will be based. Then, we briefly re- ${ }_{115}$ view the literature about the elements that govern the choice of activities.

\subsection{SimNCD: a formal model for NCDs}

SimNCD is an ABM that models individuals living within a social network and engaging in activities available in their physical environment. The practice of these activ- ${ }^{120}$ ities affects their inner state, i.e. the individual's characteristics including the health risk factors. It steers their health vulnerability to cure, acquire or maintain NCDs.

75 SimNCD serves as a tool in public health policies for the study of NCDs where the individual's behavior greatly in- ${ }^{125}$ fluences the predisposition to that disease. It is based on the approach IODA (Interaction Oriented Design of Agent simulations) [15]. In the following, we present its main 80 components.

\subsubsection{Agent families}

An agent family means its category. It is an abstract specification of an agent [15. The agents in SimNCD are either activities (e.g. take a walk, go to a party, work, and play) or individuals (children or adults).

The activities are part of the physical environment layer of the model. They are represented by the agent family $A^{a c t i v}$, whereas the individuals constitute the social network layer and are represented by the agent family $A^{\text {indiv }}$.

\section{2.1.2. Interaction}

All behaviors in SimNCD are modeled as autonomic interactions (i.e. actions and communications) and organized in an interaction matrix 11, 15. The overall functioning of an individual is as follows: among the perceived 95 activities, each individual agent chooses one to practice, considered as the goal activity (this behavior is modeled ${ }_{145}$ as an interaction $\left.I^{\text {ChooseActiv }}\right)$. Then he/she moves towards its location $\left(I^{\text {Move }}\right)$ and attempts to engage in it $\left.I^{\text {Engage }}\right)$. If the individual succeeds in doing so, he/she practices the goal activity. Finally, the individual quits the goal activity $\left(I^{\text {Quit }}\right)$.

During the practice of the activity, the individual can exhibit different kinds of behaviors. SimNCD models them as instances of three abstract interactions that are to be instantiated when studying a specific NCD:

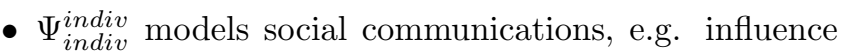
of friendship of the smoking behavior [16, 17.

- $\Psi_{\text {activ }}^{\text {indiv }}$ describes an interaction with the physical environment, such as perceiving or modifying it, e.g. the influence of the environment on the walking behavior [9. 18, 19.

- $\Psi_{\emptyset}^{\text {indiv }}$ updates the individual's inner state, which is the health risk factors, e.g. influence of the physical activity on childhood obesity [11, 20].

All the interactions are presented in the interaction matrix (see Table 1), where each one is assigned to the corresponding source agent family that initiates the behavior, and the target agent family that is affected by it.

\subsubsection{Health risk factors}

SimNCD allows a thorough study of the evolution of the health based on dynamic personal and contextual factors that are directly or indirectly predisposing to NCDs. It describes an individual's inner state based on health risk factors, like $f^{\text {energy intake }}$ or $f^{\text {smoking behavior }}$. These factors can be biological, psychological, social, behavioral, factors from the physical environment, etc. [2, 5, 11, 21]. They influence each other via updates that can occur every time the individual practices an activity. An update is described as $\Psi_{\text {set of target factors }}^{\text {set } \text { of } \text {. }}$.

For instance, we can raise the cholesterol level, update the weight, change the mood, or renew friendship ties. In order to implement said updates, the designer adds an interaction that instantiates one of the three abstract behaviors $\Psi_{\text {indiv }}^{\text {indiv }}, \Psi_{\text {activ }}^{\text {indiv }}$, and $\Psi_{\emptyset}^{i n d i v}$. For example, if we wish to model the influence of friendship (source factor) over the consumption of alcohol (target factor), we could declare two risk factors falcohol consumption and $f$ friendship, then we add an interaction $\Psi_{f^{\text {alcohol consumption }}}^{f_{\text {friendship }}}$. This latter could inherit from the social abstract interaction $\Psi_{\text {indiv }}^{\text {indiv }}$.

\subsection{Modeling agents in the study of NCDs}

Agents in ABMs are often equipped with human reasoning mechanisms that allow them to understand and react to the changes occurring in their environment. Their modeling is based on information extracted from the social and physical environments, as well as mental attitudes, e.g. desires, beliefs, preferences, trust and reputation [12, 13, 14.

We focus on modeling agents in the context of NCDs. The literature proposes several models with different included risk factors, as well as diverse study objectives. Table 3 illustrates some examples from the literature. It describes the cognitive data and reasoning processes on which the proposed models are based. 
Table 1: The behaviors in SimNCD are initiated by individuals $\left(A^{\text {indiv }}\right)$. They are modeled as a set of interactions $\left(I^{x}\right)$ and abstract updates $\left(\Psi_{\text {set of target factors }}^{\text {set }}\right)$. They affect themselves $(\emptyset)$, other individuals $\left(A^{\text {indiv }}\right)$, or the physical environment $\left(A^{\text {activ }}\right)$ 11]

\begin{tabular}{lllll}
\hline \multirow{2}{*}{ Source agent family } & \multicolumn{4}{l}{ Target agent family } \\
\cline { 2 - 5 } & $\emptyset$ & $A^{\text {indiv }}$ & $A^{\text {activ }}$ \\
\hline \multirow{2}{*}{$A^{\text {indiv }}$} & Interactions & $I^{\text {ChooseActiv }}$ & - & $I^{\text {Engage }}$ \\
& & $I^{\text {Move }}$ & - & $I^{\text {Quit }}$ \\
\cline { 2 - 5 } & Abstract updates & $\Psi_{\emptyset}^{\text {indiv }}$ & $\Psi_{\text {indiv }}^{\text {indiv }}$ & $\Psi_{\text {activ }}^{\text {indiv }}$ \\
\hline$A^{\text {activ }}$ & & - & - & - \\
\hline
\end{tabular}

The cognitive data are basically thresholds and measures that describe a subjective point of view of an indisuch as preferences or attitudes $[26,36]$. The proposed models often include the same concepts of mental attitudes. Yet these latter are mostly modeled differently depending on the context and the knowledge/data available to the study. For instance, both ABMs [9, 18, 19] model 19 it is calculated based on - among other data - the configuration of the streets in the neighborhood, whilst in 18 it is calculated based on a function of the individual's age and a random component, and in [9] the decision utility function takes into account the person's socioeconomic status and the time needed to arrive at the destination.

As for the reasoning processes, they depict the decisionmaking mechanism. They can be as simple as probabilistic and rule-based decisions [22, 23, or based on a more 200 complicated reasoning, such as utility functions [9, 24, 25. They rely on other information, like the taste preference [26] and the distance to school 27.

In our context, we aim to extend SimNCD with a reasoning mechanism in order to allow the simulated individuals ${ }^{205}$ 175 to choose the activities they perform. As shown in Table 3 the literature proposes several models in which the individuals make a decision about their destination and move towards it [18, 19, 23, 24, 26, 27, 28. The reasoning process in these models relies on data that are strongly ${ }^{210}$ dependent on the context and hypothesis of each study. Nevertheless, SimNCD is not specific to a particular disease or hypothesis. Therefore, we refer to the literature in order to find the elements of data that are necessary for the decision-making independently of the studied NCD.

The literature exposes the elements that arbitrate the ${ }_{215}$ choice of activities. These elements are mostly measured via questionnaires [29, 30, 31, that include a number of items. Each item has a set of possible scores.

For instance, the Preferences for Activities for Children questionnaire (PAC 29, see Table 2) is usually used for 55 activities, represented by cards that each child fills, e.g. the child specifies with whom he/she practices the activity. Then, statistics can be derived on, for example, the preference for a type of activities [32].

195 In the next section, we propose a solution for a reasoning process that uses these data in order to decide on the 225 activity to practice.
Table 2: An extract from the questionnaire Preferences for Activities for Children (PAC) 29]

\begin{tabular}{ll}
\hline Items & Scores \\
\hline Domain & $\begin{array}{l}\text { Formal (organized, e.g. perform sports) or } \\
\text { informal (e.g. gardening) }\end{array}$ \\
\hline Type & $\begin{array}{l}\text { Recreational, physically active, social, skill- } \\
\text { based or self-improvement }\end{array}$ \\
\hline With & Alone, close family, other relatives, friends, \\
whom? & others \\
\hline Where? & Home, relatives home, neighborhood, \\
& school, in community, beyond community \\
\hline
\end{tabular}

\section{Model design}

We propose a reasoning mechanism as an extension to SimNCD that helps an individual select the activity that seems the most advantageous from his/her point of view. We express this point of view using preferences. As future work, other mental attitudes could be considered.

We model this mechanism in three phases. First, in the initialization phase, the person's preferences and the characteristics of the activities are described. Next, the person compares the characteristics of the candidate activities and his/her preferences. In this phase, the individual assigns evaluations to the candidate activities, hence the name: evaluation phase. Finally, the person selects the best activity suited to his/her liking in the selection phase.

\subsection{Initialization phase}

This phase is executed only once at the beginning of the simulation. It formalizes how (i) the individuals express their preferences and (ii) the activities describe their characteristics. Both steps are based on the same set of criteria.

\subsubsection{Decision criteria}

We extract the decision-making criteria from the questionnaires that are proposed by the literature. Let this set be $C=\left\{C_{j}\right\}$, where $j \in[1, n]$ and $\mathrm{n}$ is the number of criteria. The choice of the questionnaire can be done by the public health expert who's conducting the study. In this paper, we will base our examples on Table 1, in which case the obtained criteria would be: $C=\left\{C_{1}=\right.$ domain, $C_{2}=$ type, $C_{3}=$ social context, $C_{4}=$ physical context $\}$. 


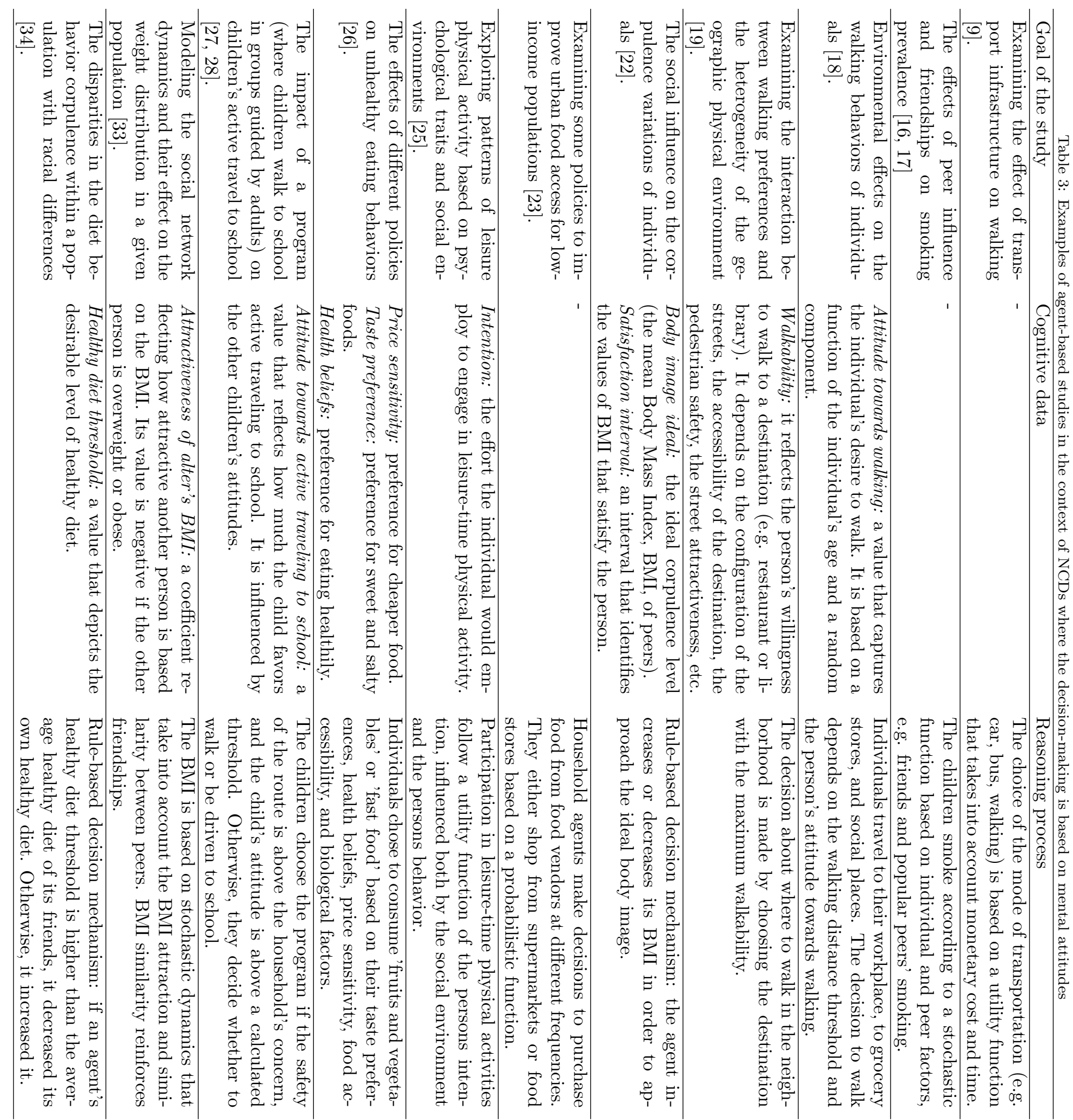


We consider the scores of the criteria to be sub-criteria. Hence, each criterion $C_{j}$ is a set of sub-criteria: $C_{j}=$ $\left\{x_{j u}\right\}$, where $x_{j u}$ is a linguistic term and $u \in\left[1,\left|C_{j}\right|\right]$. For example, $C_{1}=\left\{x_{11}=\right.$ formal, $x_{12}=$ informal $\}$ and $C_{3}=\left\{x_{31}=\right.$ alone, $x_{32}=$ close family, $x_{33}=$ other relatives, $x_{34}=$ friends, $x_{35}=$ other $\}$.

We now describe how the activities and the individuals use these items to model their characteristics and preferences.

\subsubsection{Activity characteristics}

Each activity describes its own characteristics according ${ }^{280}$ to the set of criteria $\mathrm{C}$. We notice that some criteria can have more than one value. In Table 2 , for instance, an acpeople.

We formally describe the characteristics of an activity $\beta_{i}{ }^{285}$ as: $\kappa^{\beta_{i}}=\left\{\left\langle C_{j}, \kappa_{j}^{\beta_{i}}\right\rangle\right\}$, where $j \in[1, n] . \kappa_{j}^{\beta_{i}}$ is the description of $\beta_{i}$ regarding the criterion $C_{j} \in C$. It is a set of linguistic terms: $\kappa_{j}^{\beta_{i}}=\left\{x_{j u}^{\beta_{i}}\right\}$, where $x_{j u}^{\beta_{i}} \in C_{j}, u \in\left[1,\left|C_{j}\right|\right]$, and by consequence, $\kappa_{j}^{\beta_{i}} \subset C_{j}$. The fact that a characteristic $\kappa_{j}^{\beta_{i}}$ is a set covers the cases where more than one value can be attributed to a criterion. For instance, $\beta_{1}=$ "Perform martial arts" can be described with ${ }_{250} C_{3}=$ "social context" as: $\kappa_{3}^{\beta_{1}}=\left\{x_{31}^{\beta_{1}}=\right.$ alone, $x_{34}^{\beta_{1}}=$ friends, $x_{35}^{\beta_{1}}=$ others $\}$.

\subsubsection{Individual preferences}

Regarding preferences, we consider them as a psychological factor $f^{\text {Prefs }}$ that assigns a likeness degree in a ${ }_{255}$ Likert scale to each sub-criterion. Let $L_{M}$ be a Likert scale of $\mathrm{M}$ terms. For $M=3, L_{3}=\left\{\tau_{1}=\right.$ not like, $\tau_{2}=$ like, $\tau_{3}=$ really like $\}$. Therefore, to each sub-criterion $x_{j u}$, the individual assigns a linguistic term $\tau_{i} \in L_{M}$. We propose to describe the preferences as follows: $f^{\text {Prefs }}=$ $\left\{\left\langle C_{j}, x_{j u}, \tau_{j u}\right\rangle\right\}$, where $\tau_{j u} \in L_{M}$ expresses the individual's likeness towards $x_{j u}$ of the criterion $C_{j} \in C, u \in\left[1,\left|C_{j}\right|\right]$ and $j \in[1, n]$. Hence, $f^{\text {Prefs }}$ includes as many elements as sub-criteria: $\left|f^{\text {Prefs }}\right|=\sum_{j=1}^{n}\left|C_{j}\right|$. For example, $f^{\text {Prefs }}=$ $\left\{\left\langle C_{1}, x_{11}=\right.\right.$ formal, $\tau_{11}=$ really like $\rangle,\left\langle C_{1}, x_{12}=\right.$ 265 informal, $\tau_{12}=$ like $\rangle,\left\langle C_{2}, x_{21}=\right.$ recreational, $\tau_{21}=$ not like $\rangle, \quad\left\langle C_{2}, \quad x_{22}=\right.$ physically active, $\tau_{22}=$ really like $\rangle,\left\langle C_{2}, x_{23}=\right.$ social, $\tau_{23}=$ not like $\left.\rangle, \ldots\right\}$.

\subsection{Evaluation phase}

Unlike the initialization phase, the evaluation phase and

the selection phase are executed when an individual agent decides on the activity to practice. In the evaluation phase, a person uses the elements that we set previously in order to evaluate the candidate activities. The output of this phase is composed of evaluation sets for each activity.

In fact, the candidates are the perceived activities $\beta_{i}$. For each $\beta_{i}$, the person examines the characteristics $\kappa_{j}^{\beta_{i}}$ one by one, and assigns the corresponding likeness from the preferences $f^{\text {Prefs }}$, as represented in Figure 1.

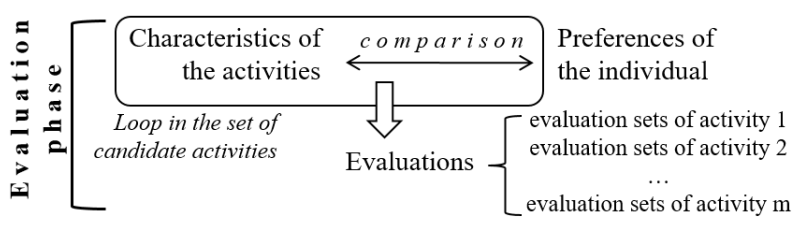

Figure 1: Evaluation phase

\subsubsection{Formal description}

We obtain a set of evaluations $E^{\beta_{i}}$ that can be described as a relationship between the set of criteria $\mathrm{C}$ and the linguistic terms from $L_{M}$. These linguistic terms are not necessarily ordered because we intend to aggregate them later in order to obtain one value describing the performance of each activity (the aggregation is part of the selection phase). Therefore, we omit the sub-criteria from $E^{\beta_{i}}$. In other words, $E^{\beta_{i}}$ describes the likeness levels to each criterion, e.g. $\left\langle C_{3},\{\right.$ really like, like, like $\left.\}\right\rangle$, instead of $\left\langle C_{3},\left\{\left\{x_{31}^{\beta_{1}}=\right.\right.\right.$ alone, really like $\},\left\{x_{34}^{\beta_{1}}=\right.$ friends, like $\},\left\{x_{35}^{\beta_{1}}\right\}=$ others, like $\left.\left.\}\right\}\right\rangle$. We describe a set of evaluations as: $E^{\beta_{i}}=\left\{\left\langle C_{j}, e_{j}^{\beta_{i}}\right\rangle\right\}, j \in[1, n] . e_{j}^{\beta_{i}}$ is a set of linguistic terms from $L_{M}$ that describe the preferences towards all the sub-criteria of the criterion $C_{j}$, retrieved from the characteristics of $\beta_{i}$. Hence, $e_{j}^{\beta_{i}}=\left\{\tau_{j u}\right\}$, where $\exists\left\langle C_{j}, x_{j u}, \tau_{j u}\right\rangle \in f^{\text {Prefs }}, \tau \in \tau_{j u} \in L_{M}$, the sub-criterion $x_{j u}=x_{j u}^{\beta_{i}} \in \kappa_{j}^{\beta_{i}}$ with $\left\langle C_{j}, \kappa_{j}^{\beta_{i}}\right\rangle \in \kappa^{\beta_{i}}$ (i.e. $x_{j u}^{\beta_{i}}$ ) is part of the characteristics $\kappa^{\beta_{i}}$ of $\beta_{i}$ ). The terms of $e_{j}^{\beta_{i}}$ are not necessarily ordered, distinct, balanced, nor different.

\subsubsection{Algorithm}

In Algorithm 1, we present EvaluateActivity that constructs the set of evaluations of $E^{\beta_{i}}$ of an activity $\beta_{i}$.

$\overline{\text { Algorithm } 1 \text { EvaluateActivity }\left(\kappa^{\beta_{i}}, f^{\text {Prefs }}\right) \text { calculates }}$ the evaluation of an activity

Require: $\kappa^{\beta_{i}}$ : the set of characteristics of the activity $\beta_{i}$; $\kappa^{\beta_{i}}=\left\{\left\langle C_{j}, \kappa_{j}^{\beta_{i}}\right\rangle\right\}, j \in[1, n], \kappa_{j}^{\beta_{i}}=\left\{x_{j u}^{\beta_{i}}\right\}$

$f^{\text {Prefs }}$ : the set of preferences of the individual; $f^{\text {Prefs }}=$ $\left\{\left\langle C_{j}, x_{j u}, \tau_{j u}\right\rangle\right\}, \tau_{j u} \in L_{3}$, and the sub-criterion $x_{j u} \in$ $C_{j}, u \in\left[1,\left|C_{j}\right|\right]$ and $j \in[1, n]$

\section{Ensure: $E^{\beta}$}

initialize $E^{\beta_{i}}$ to an empty list

for criterion $C_{j} \in C$ do

initialize $e_{j}^{\beta_{i}}$ to an empty list

for sub-criterion $x_{j u}^{\beta_{i}}$ in $\kappa_{j}^{\beta_{i}}$ do

add $\left\{\tau_{j u} \mid \exists\left\langle C_{j}, x_{j u}, \tau_{j u}\right\rangle \in f^{\text {Prefs }}\right.$, and $\left.x_{j u}=x_{j u}^{\beta_{i}}\right\}$ to $e_{j}^{\beta_{i}}$

end for

add $\left\{\left\langle C_{j}, e_{j}^{\beta_{i}}\right\rangle\right\}$ to $E^{\beta_{i}}$

end for 


\subsection{Selection phase}

Once the evaluations are prepared, the individual agent selects the activity to practice. First, it aggregates the sets of evaluations for each activity, and then compares them in order to choose the preferred one. Since the evaluations ${ }^{360}$ are based on criteria with discrete evaluations (linguistic) and a finite number of solutions, we are facing a multiattribute decision-making (MADM) problem [35, 36, 37.

\subsubsection{Multi-attribute decision-making methods}

The MADM allow the selection and classification of alternatives. They can be classified in four categories: elementary methods (e.g. Maximin), outranking methods (e.g. ELECTRE and PROMETHEE), utility methods (e.g. SMART and AHP) and others (e.g. SAW and TOPSIS) [35, 36, 37. The choice of the method to adopt depends greatly on the available information regarding the criteria. Hence, we do not limit such choice in our model. An example will be illustrated in the case study.

\subsubsection{Linguistic representation and aggregation}

We look into how to represent and aggregate the information in the evaluations. In our decision-making problem, the evaluations are sets of linguistic terms: $e_{j}^{\beta_{i}}=$ $\left\{\tau_{j u}\right\}, \tau_{j u} \in L_{M}$.

The MADM methods are usually applied to evaluations and not sets of evaluations. Therefore, we propose to aggregate each set of linguistic terms $e_{j}^{\beta_{i}}$ into one value $\tilde{e}_{j}^{\beta_{i}}$.

The multi-valued logic 38 brings a solution to this matter by providing a representation of vague, uncertain, or 370 imprecise knowledge using the human language. It associates a symbolic representation with linguistic terms and allows to express the intermediary degrees of truth between the true and the false. For a given concept, the degrees of truth can be represented as a set: $L_{k}=$ $\left\{\tau_{1}, \tau_{2}, \ldots, \tau_{k}\right\}, k \geq 2$ and $\forall i \geq 2, \tau_{i} \leq \tau_{i+1}$. In our context, 375 we use the multi-valued logic to represent the evaluations that are expressed with linguistic terms in $L_{M}$.

We examine the linguistic aggregation operators (LAOs) proposed in the literature. They can be classified in four categories 39]: the LAOs based on a linear order [40, 41, the LAOs based on the extension principle [42], the $\mathrm{LAO}_{380}$ based on symbols [43, and the LAOs based on continuous linguistic domain [39, 44]. The LAOs in the first three categories allow approximating the result of an aggregation. However, the fourth category produces an aggregation result that is more precise on a continuous scale. Thus, we choose the fourth category that includes the linguistic 2-385 tuple representation 44 and the method of virtual terms 39. Both these methods extend the original discrete linguistic domain to a continuous linguistic set. The main difference between them is that the virtual terms method can return a linguistic term that doesn't belong to the orig-390 inal set. We opt for the linguistic 2-tuple representation 44]. In this numeric-symbolic model, a data is a linguis- where $\mathrm{s}$ is a term from the original discrete linguistic set, and $\gamma$ is a numeric value expressing a translation degree from the term s. Thus, $\gamma$ indicates how far $l^{2 t}$ is from s. If $l^{2 t}$ coincides with $\mathrm{s}$, then $\gamma=0$, otherwise $\gamma \in[-0.5,0.5)$. This method proposes some LAOs, such as the weighted average and the induced weighted average [44.

\subsubsection{Formal description}

The input of the selection phase is the sets of evaluations $e_{j}^{\beta_{i}}$ per activity $\beta_{i}$ and per criterion $C_{j}$. We present them in a matrix (a line per activity and a column per criterion):

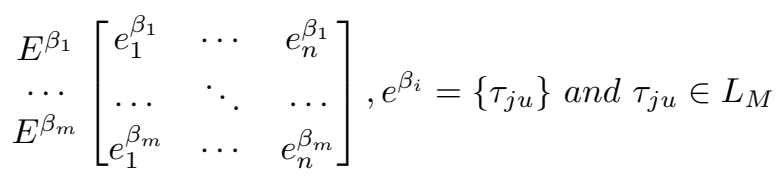

In this phase, we proceed as follows:

- We aggregate each $e_{j}^{\beta_{i}}$ into one 2-tuple linguistic term (e.g. using the 2-tuple arithmetic average [44]). We obtain the 2-tuple matrix:

$$
\begin{gathered}
\tilde{E}^{\beta_{1}} \\
\cdots \\
\tilde{E}^{\beta_{m}}
\end{gathered}\left[\begin{array}{ccc}
\tilde{e}_{1}^{\beta_{1}} & \cdots & \tilde{e}_{n}^{\beta_{1}} \\
\cdots & \ddots & \cdots \\
\tilde{e}_{1}^{\beta_{m}} & \cdots & \tilde{e}_{n}^{\beta_{m}}
\end{array}\right], \tilde{e}^{\beta_{i}}=\left(\tau_{j}^{\beta_{i}}, \gamma_{j}^{\beta_{i}}\right)
$$

- We use a MADM method, adapted and applied for the 2-tuple representation, in order to calculate the performance of each activity $\beta_{i}$.

- We select the activity with the best performance.

\subsection{Case study: childhood obesity}

In 11, 45, the authors proposed an ABM to simulate the children's physical activity and its relationship with obesity, called $\operatorname{SimNCD}{ }^{C h O}$ (SimNCD for Childhood Obesity). We briefly present this ABM, and then we extend it with our proposed reasoning process.

\subsubsection{Context of the case study}

SimNCD $D^{C h O}$ [11, 45] models children who are engaged in activities that affect their health regarding acquiring or maintaining obesity. The included risk factors are:

- Biological factors: Age and gender.

- Physical environment: The opportunity to practice physical activity. This factor reflects the amount of Moderate to Vigorous Physical Activity (MVPA) offered by each activity. For instance, the opportunity of "playing at a park" is bigger than that of "playing video games". This factor is described as a percentage, and the sum of the opportunities of the activities practiced by a child in a physical environment that is favorable for a good health is $100 \%$. The more such sum increases above $100 \%$, the more the physical environment of the child is highly favorable, and the less it is, the more the physical environment is unfavorable. 
- Mental attitude: The attitude towards an activity is a value in $[0,2]$. This factor is inspired by the literature, such as the attitude towards active traveling to school $_{450}$ 28] and the attitude towards drinking 46]. It reflects how far the child is motivated to take advantage of the opportunity that is offered by an activity. The higher the value of this coefficient, the more the child is considered to be active. This factor can be interpreted as unmotivated, motivated, or highly motivated.

- Behavioral factor: The physical activity, measured in daily minutes of MVPA. It can be interpreted as inactive, active or highly active. It follows the normal course of the children's physical activity via a function called basicMVPA(age,gender). This function pro-460 vides the amount of MVPA minutes each child would be engaged on account for the normal course of MVPA (more details can be found in [1, 11]). The physical activity also depends on the encountered opportunities $\left(o p p^{\beta_{i}}\right)$ and the child's attitude towards the activity $\beta_{i}$ (attitude $\left.{ }^{\beta_{i}}\right)$. By practicing $\beta_{i}$, the child gathers $M V P A^{\beta_{i}}$, calculated as follows: $M V P A^{\beta_{i}}=$ opp $^{\beta_{i}} *$ attitude ${ }^{\beta_{i}} *$ basicMVPA(age,gender $)$. The amount of MVPA gathered during a day is the sum of $M V P A^{\beta_{i}}$ of all the activities practiced during that day.

- Physiological factor: The corpulence, measured in Body Mass Index (BMI) and characterized as normal, overweight or obese. It follows the normal trends of development of the children's BMI as described in the children's growth references [47. The corpulence factor is updated depending on the average amount of daily MVPA practiced over the year. The effect of the daily MVPA on the child's BMI was aggregated from results offered by the literature. This function is presented in details in 11.

This case study was validated against empirical data d65 $_{4}$ extracted from interventions 11. A public health intervention is a set of actions involving a group of people. It aims to make changes to prevent or treat a disease [1].

\subsubsection{Modeling the choice of the goal activity}

We extend $\operatorname{SimNCD} D^{C h O}$ in order to include the proposed reasoning process. This latter interferes on two lev- ${ }^{470}$ els in our ABM. On the first level, it steers the child's behavior by allowing him/her to choose an activity. We use the questionnaire PAC for the decision criteria (Table 2). Besides that, in the selection phase, we use the MADM method TOPSIS (Technique for Order of Preference by Similarity to Ideal Solution), applied for the linguistic 2tuple [37, 48, 49. TOPSIS chooses the alternatives that ${ }_{475}$ are simultaneously the most advantageous and least costly. This is achieved by calculating a Relative Proximity coefficient $\in[0,1]$, for each alternative.

This measure represents a subjective aggregated evaluation of an activity from the child's point of view. We refer to it as the function RelProx ${ }^{2 t}$. The selected activity is the one with the highest Relative Proximity value. This phase is summarized in Algorithm 2 .

\subsubsection{Modeling the effects of the preferences on the health}

On the second level, the preferences affect the way the child practices the activities via the risk factor "attitude towards an activity". In fact, we suppose that a child takes as much advantage of an activity as the preference for this activity. In other words, a child who prefers an activity has a positive attitude towards it. We already have a coefficient that reflects the performance of each activity, which is the Relative Proximity. We use it to calculate the child's attitude: attitude $\beta_{i}=\operatorname{RelProx} \operatorname{Pr}^{2 t}\left(\tilde{E}^{\beta_{i}}\right) * 2$, where $\tilde{E}^{\beta_{i}}$ is the vector of aggregated 2-tuple evaluations of the activity $\beta_{i}$. Since attitude $\beta_{i} \in[0,2]$ and $\operatorname{RelProx}^{2 t} \in[0,1]$, this latter is multiplied by 2 .

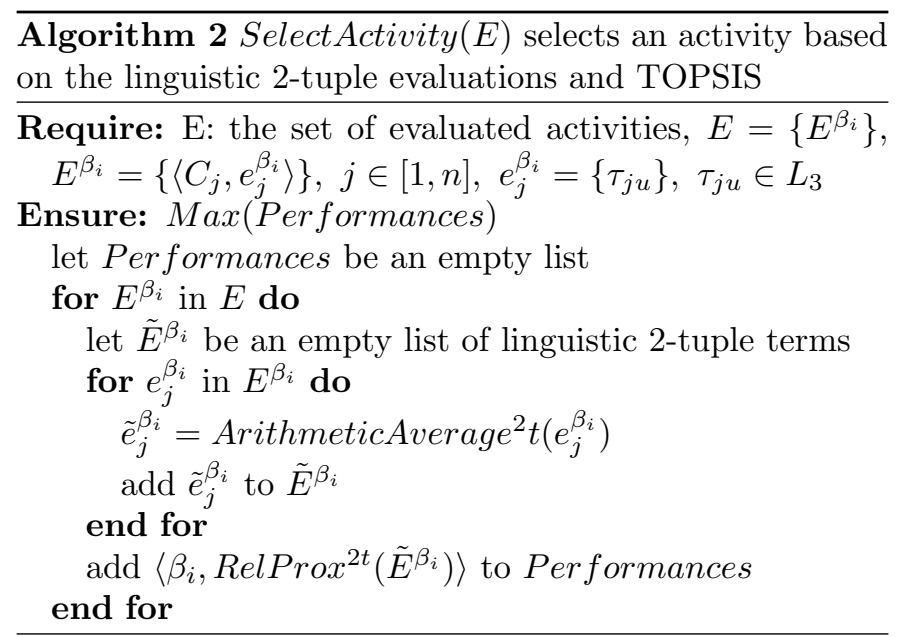

\subsubsection{Summary of the child's behavior}

The overall behavior of a child agent can be summarized as a loop of the following steps:

- Choosing the activity to practice:

- Evaluating the activities based on their characteristics and the preferences.

- Deciding on the activity to practices.

- Moving towards the chosen activity and practicing it.

- Updating the child's inner state:

- Updating the age risk factor.

- Calculating the attitude towards the activity.

- Updating the MVPA based on the age, the gender, the opportunity to MVPA, and the attitude.

- Updating the BMI based on the age, the gender, the current BMI, and the MVPA.

- Quitting the activity. 


\section{Results and discussion}

We implemented $\operatorname{SimNCD} D^{C h O}$ with the framework JEDI [15] with an option to activate the reasoning process. We use this simulator to validate our ABM against empirical data. Then, we run it in different scenarios in ${ }_{540}$ order to predict insights about real children's corpulence variations.

\subsection{Simulation for validation}

Finding detailed data to help evaluate detailed predic-545 tion models such as SimNCD can be quite challenging 11.

${ }_{490}$ Among other reasons, that is due to the fact that the field studies proposed by the epidemiological literature rarely include the same contexts, hypotheses, and factors.

To evaluate our model, we replicated the findings of field ${ }_{550}$ studies 50, 51, 52, 53 and compared the simulated results with the actual ones. The results obtained with the interventions [51, 50, 53] resemble those presented in [11. In this paper, we detail the results obtained for the intervention of $\mathrm{Li}$ et al. [52].

We note that, in the validation, we do not focus on We effect of preferences on the outcome of the simulations. We rather examine the overall results of our model to compare them with those reported from empirical studies. A discussion about the children's weight statuses specified by their preferences will be detailed in section $505 \quad 4.2$.

The physical activity intervention 52 includes a population of 4700 children, assigned randomly to the control group (that did not follow a physical activity program) or the intervention group. This latter experienced an addi-

510 tional daily $20 \mathrm{~min}$ of MVPA at school. The study observes and compares the development of children's corpulence in both groups.

In our simulation, we included the following risk factors: age, gender, opportunity to MVPA (measured in percent-

515 age, with the sum of daily opportunities in a favorable physical environment is $100 \%$ ), attitude towards the activities, and adiposity (measured in BMI, $\mathrm{kg} / \mathrm{m}^{2}$ ).

We started by reproducing the data of the controlled group over one simulated year. As described in 52, the sample size of the control group was 2115 children $\left(1050^{555}\right.$ girls) of 9 years old, with a baseline BMI of $17.74 \pm$ $3.61 \mathrm{~kg} / \mathrm{m}^{2}$ (mean \pm standard deviation). Using these data, we initialized the number of children, their age, gender, and BMI. We set the preferences (by consequence, the attitude towards the practiced activities) to random. Since we didn't find information that qualify the physical560 environment in the paper, we supposed that the physical environment is favorable and we set the opportunity to MVPA to $100 \%$.

Next, we configured the simulation of the intervention group as described in [52]: 2072 children (957 girls) of $9_{565}$ years old, with a BMI of $17.79 \pm 3.61 \mathrm{~kg} / \mathrm{m}^{2}$ at baseline. The preferences were set to random. As for the opportunity to MVPA, it was set to $100 \%$ plus the opportunity given by the daily $20 \mathrm{~min} /$ day of MVPA. To transform the minutes of opportunity to a percentage, we divided them by the normal course of children's daily MVPA (i.e. corresponding to a normal health), which is $60 \mathrm{~min}$ and 72 min of MVPA for, respectively, 9 years old girls and boys 1. Thus, the opportunity to MVPA was $(100 \%+20 / 60)$ for girls and $(100 \%+20 / 72)$ for boys.

Figure 2 illustrates the results of the average BMI of both groups in the original and the simulated study. The obtained simulated effect of the intervention is $0.07 \mathrm{~kg} / \mathrm{m}^{2}$. It is calculated by subtracting the BMI of the intervention group from the BMI of the controlled group. The results show that this value is close to the effect stated by the real intervention, $0.11 \mathrm{~kg} / \mathrm{m}^{2} 52$. This suggests that our model is capable of delivering realistic indications about changes in the BMI of children. Such validation should be extended to cover all the studied age range, from 9 to 10 years old.

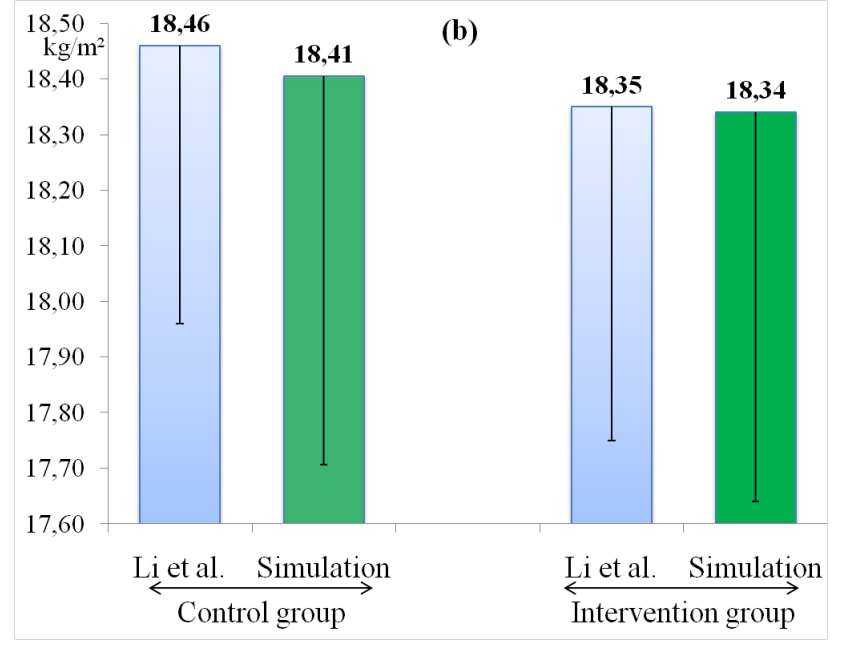

Figure 2: Comparison between the average BMI results of 52 and our simulations on a one-year scale.

\subsection{Simulation for prediction}

The presented reasoning mechanism allows simulating children with different preferences about the activities they practice in their daily lives. Such simulation would help researchers and health practitioners obtain insights about the development of the corpulence of real children with different preferences and physical activity behaviors.

\subsubsection{Experimental design}

The literature 54 suggests that the preferences of children are different from the preferences of adolescents. In this paper, we did not take into account the evolvement of the preferences with time. Therefore, we chose to simulate the development of the children during primary school (initialized at 6 years-old and simulated until 10 years-old). Hence, the results were gathered after 4 years when the children reached 10 . The simulated characteristics are: 
- Population: 1000 boys and 1000 girls.

- Different opportunities to MVPA offered by three activities:

- Stay at home: a sedentary activity (such as play- ${ }^{625}$ ing video games).

- Play at the park: a moderate activity.

- Perform sports: a vigorous activity.

- The preferences of children were set randomly in $L_{3}=$ \{not like, like, really like\}.

We simulated three scenarios for both genders. The first scenario depicted children with normal BMI with a high risk to become overweight. The second scenario involved overweight children, and the third one was about obese children.

\subsubsection{Results and discussion}

The extended SimNCD allows making predictions (based on a mental attitude risk factor) than the original SimNCD model. We examined the changes in BMI in the different simulated scenarios (Figure 3) while taking into account the activities that the children perceived (i.e.645 the activities that were available to them) and the ones the initial weight status (normal, overweight or obese), the perceived and the preferred activities.

At the end of the simulation, we noticed that the chil-650 dren who stayed at home (cases 'a') had noticeably higher a striction the claim that physical activity is a key factor in the dynamics of obesity [1, 2] but also proposes a more precise quantification of 655 such relationship. For instance, overweight girls and boys who practiced sports had, respectively, up to $26 \%$ and $10 \%$ less BMI than those who remained at home.

We also noted that boys were more advantageous than girls in most simulations in terms of obesity risk. This660 suggests that overweight and obesity may be more difficult to overcome in girls than in boys, or that the relationship between PA and adiposity is stronger in boys, as suggested by the literature [47, 55].

In order to better understand the results, we computed children's BMI distribution in terms of risk at the end of the simulation, presented in Figure 4. As mentioned ear-665 lier, boys were more advantageous than girls, especially in the case of children initialized as obese, and even more for those who were overweight at baseline. However, that was not the case of the ones who had normal weight. In fact, $27 \%$ of the normal boys who practiced sedentary ac-670 tivities and $5 \%$ of those who practiced moderate activities (but did not perform vigorous activities) ended up as overweight. Nevertheless, all girls with normal weight remained as such, no matter the activity they preferred or girls being more disadvantageous than boys only applies to overweight and obese girls (we call this 'hypothesis 1').

Among the children who were initialized as overweight, girls had a much higher risk of becoming obese, especially those who were sedentary ( $93 \%$ became obese), compared to boys (only $6 \%$ became obese). Besides that, children who engaged in vigorous activities (sports) had a consistent chance at becoming normal: $52 \%$ chance for girls and $37 \%$ for boys. The overweight girls always had a risk to become obese, but the risk was lower $(5 \%)$ when they were vigorously active.

On the other hand, some of the obtained results were conflicting. For instance, in the case 'c' of overweight girls (Figure 4), 40\% of these girls had normal weight, $46 \%$ remained overweight, and $14 \%$ became obese. In our opinion, this can be explained by the fact that a given child takes advantage of practicing an activity as much as he/she likes it, i.e. via the attitude towards the activity that depends on the preferences. In some cases, even though a child performs sports, his/her corpulence might deteriorate. Thus, we can suppose that a physical activity, even a vigorous one, is not sufficient if not coupled with a positive attitude towards it ('hypothesis 2'). And since such controversy was only observed in overweight girls, we can assume that this age is quite critical for the development of their corpulence, more so than other girls/boys ('hypothesis $\left.3^{\prime}\right)$.

As for children initialized as obese, the only cases where their corpulence improved were when they practiced moderate and/or vigorous activities (cases 'c' and 'd'). Based on these results, the best chances of obese children at becoming overweight were $12 \%$ for girls and $35 \%$ for boys. These results also suggest that performing vigorous physical activities is not enough to bring 6-year-old children from obesity to normal adiposity. This could call for additional efforts to be made on other risk factors, or it might require longer than 4 years of intervention in order to achieve a safe normal weight status ('hypothesis 4').

The hypotheses 1 to 4 call for further examination. Epidemiological field studies could be undertaken in order to verify them.

\section{Conclusion}

This paper proposes a reasoning mechanism, modeled as an extension of the formal ABM SimNCD 11. This process uses an MADM method, applied to linguistic 2tuple preferences.

Basically, this decision-making mechanism has three contributions. First, it allows the individual agents to reason and choose the best activity to practice. Secondly, it allows modeling the (in)direct effects of mental attitudes (in this case, the preferences) on a population's health. Thirdly, this work illustrates the use of SimNCD and its extensibility to include different kinds of risk factors.

We applied our solution in the study of childhood obesity and we extended the model $\operatorname{Sim} N C D^{C h O}$ [11. We 


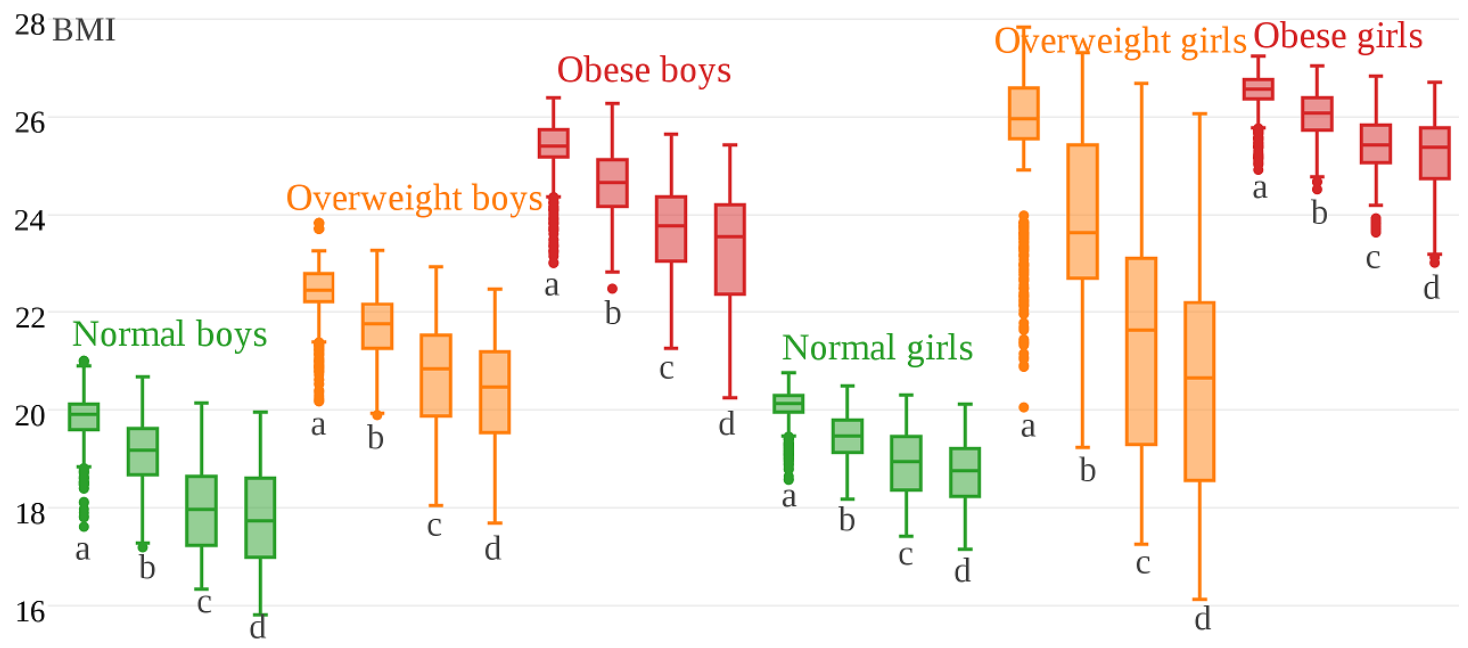

Figure 3: BMI results. The boys/girls were initialized as normal, overweight, and obese, with the preferences: a=stay at home; b=can stay at home or play at the park, and prefer playing at the park; $c=$ can stay at home or perform sports, and prefer performing sports; $d=$ can stay at home, play at the park, or perform sports, and prefer performing sports.
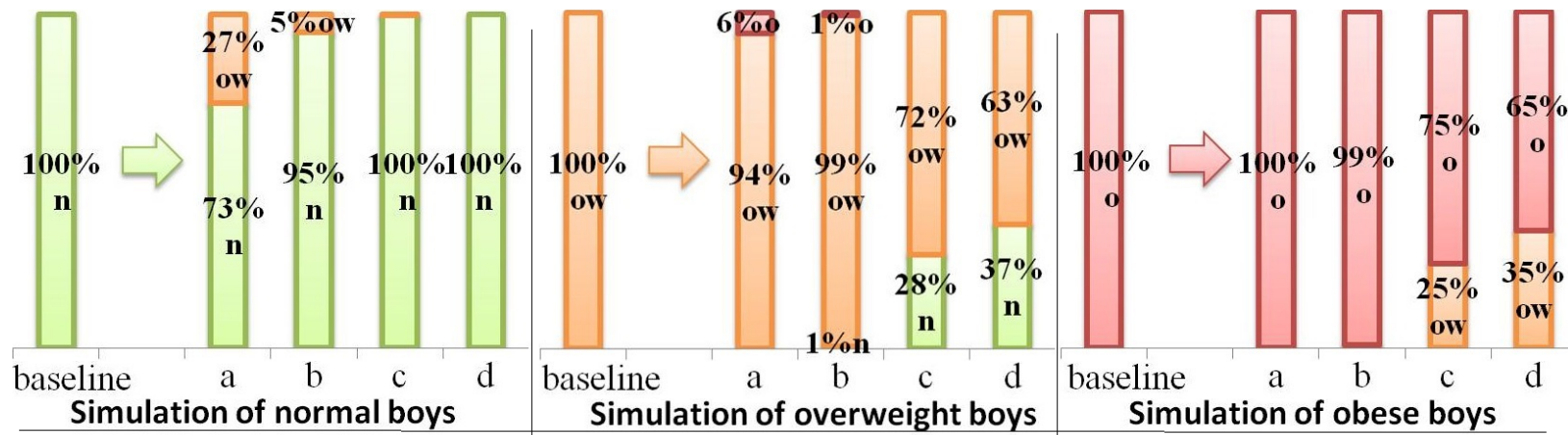

Simulation of overweight boys
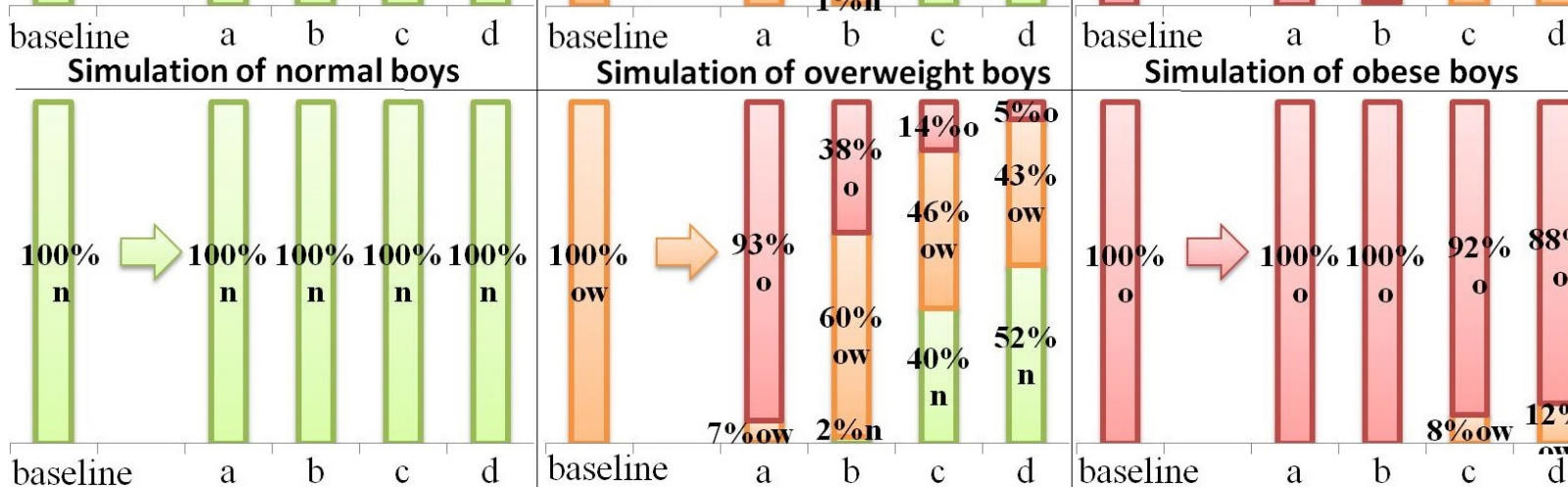

Simulation of obese boys

Simulation of normal girls

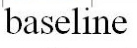

Simulation of overweight girls

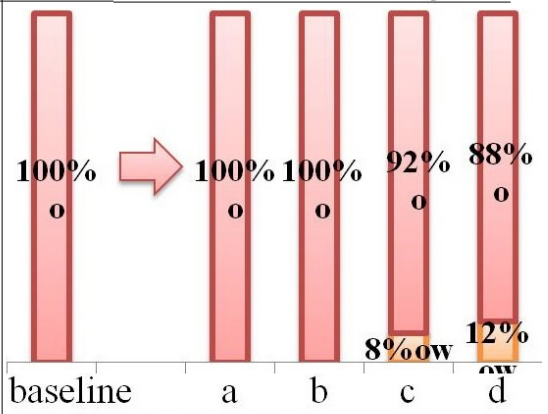

Figure 4: Distribution of the corpulence prevalence based on the BMI growth references [47. In each scenario, all children have the same corpulence status at baseline (prevalence is 100\%). Boys/girls were initialized as normal, overweight, and obese, with the preferences: a=stay at home; $b=$ can stay at home or play at the park, and prefer playing at the park; $c=$ can stay at home or perform sports, and prefer performing sports; $\mathrm{d}=$ can stay at home, play at the park, or perform sports, and prefer performing sports.

validated our system against empirical data. Then we used it to predict the indirect effects of children's preferences on the changes in their corpulence as mediated by physical activity behaviors. This system could allow the public researchers and practitioners to simulate and examine the effect of the interplay between available activities in a physical environment and children's personal preferences on their health, as exemplified by the weight status in the present study. Furthermore, the simulations that we could bring more understanding of the complex dynamics of obesity.

As future work, the proposed cognitive mechanism should be tested in other domains. It could also be extended to take into account temporal changes in the preferences, or to include other mental attitudes in the reasoning process, such as beliefs and emotions. 


\section{References}

[1] B. Guinhouya, Physical activity during the development of the child, Lavoisier, Paris, 2012.

[2] WHO, Global Status Report On Noncommunicable Diseases 2014, Tech. rep., World Health Organization (2014).

[3] Y. fan Zhang, L. Gou, T. shu Zhou, D. nan Lin, J. Zheng, Y. Li, J. song Li, An ontology-based approach to patient followup assessment for continuous and personalized chronic disease management, Journal of Biomedical Informatics 72 (2017) 4559. doi:10.1016/j.jbi.2017.06.021

[4] R. Aziza, A. Borgi, H. Zgaya, B. Guinhouya, Simulating Complex Systems - Complex System Theories, Their Behavioural Characteristics and Their Simulation, in: Proceedings of the 8th International Conference on Agents and Artificial Intelligence (ICAART), SCITEPRESS - Science and and Technology Publications, 2016, pp. 298-305. doi:10.5220/0005684602980305 780

[5] R. a. Nianogo, O. a. Arah, Agent-Based Modeling of Noncommunicable Diseases: A Systematic Review, American Journal I] of Public Health 105 (3) (2015) e20-e31. doi:10.2105/AJPH. 2014.302426

[6] Y. Li, M. A. Lawley, D. S. Siscovick, D. Zhang, J. A. Pagan, Agent-Based Modeling of Chronic Diseases : A Narrative Review and Future, Preventing Chronic Disease 13 (E69) (2016) $1-8$.

[7] M. Tracy, M. Cerdá, K. M. Keyes, Agent-Based Modeling in Public Health: Current Applications and Future Directions., 790 Annual review of public health 39 (January) (2018) 77-94. doi : 10.1146/annurev-publhealth-040617-014317

[8] C. E. Vincenot, How new concepts become universal scientific approaches: Insights from citation network analysis of agentbased complex systems science, Proceedings of the Royal Society B: Biological Sciences 285 (1874). doi:10.1098/rspb.2017. 2360

[9] P. D. Lemoine, J. M. Cordovez, J. M. Zambrano, O. L. Sarmiento, J. D. Meisel, J. A. Valdivia, R. Zarama, Using agent based modeling to assess the effect of increased Bus Rapid Tran- ${ }_{800}$ sit system infrastructure on walking for transportation, Preven-

730 tive Medicine 88 (2016) 39-45. doi:10.1016/j.ypmed.2016.03. 015

[10] R. A. Nianogo, M. C. Wang, A. Wang, T. Z. Nobari, C. M. Crespi, S. E. Whaley, O. A. Arah, Projecting the impact of hypothetical early life interventions on adiposity in children living in low-income households, Pediatric Obesity 12 (5) (2017) 398-405. doi:10.1111/ijpo.12157

[11] R. Aziza, A. Borgi, H. Zgaya, B. Guinhouya, SimNCD: An agent-based formalism for the study of noncommunicable dis- ${ }_{810}$ eases, Engineering Applications of Artificial Intelligence 52 (2016) 235-247. doi:10.1016/j.engappai.2016.03.005

[12] D. Liu, L. Schubert, Toward Self-Motivated, Cognitive, Continually Planning Agents, Computational Intelligence 31 (3) (2015) 385-417. doi:10.1111/coin.12029

[13] S. J. Russell, P. Norvig, Artificial Intelligence: A Modern Approach, Always learning, Pearson, 2013.

[14] M. Wooldridge, An Introduction to MultiAgent Systems, Wiley, 2009.

[15] Y. Kubera, P. Mathieu, S. Picault, IODA: an interaction- ${ }_{820}$ oriented approach for multi-agent based simulations, Autonomous Agents and Multi-Agent Systems 23 (3) (2011) 303343. doi:10.1007/s10458-010-9164-z

[16] D. R. Schaefer, S. A. Haas, N. J. Bishop, A dynamic model of US adolescents' smoking and friendship networks, American ${ }_{825}$ Journal of Public Health 102 (6) (2012) 12-18. doi:10.2105/ AJPH. 2012.300705

[17] D. R. Schaefer, J. Adams, S. A. Haas, Social networks and smoking: exploring the effects of peer influence and smoker popularity through simulations, Health Education \& Behavior $40\left(1_{830}\right.$ Suppl) (2013) 24S-32S. doi:10.1177/1090198113493091.

[18] Y. Yang, A. V. Diez Roux, A. H. Auchincloss, D. A. Rodriguez, D. G. Brown, A spatial agent-based model for the simulation of adults' daily walking within a city, American
II Journal of Preventive Medicine 40 (3) (2011) 353-361. doi: 10.1016/j.amepre.2010.11.017.

[19] L. Yin, Assessing Walkability in the City of Buffalo : Application of Agent-Based Simulation, Journal of urban Planning and Development 139 (3) (2013) 166-175. doi:10.1061/(ASCE)UP. 1943-5444.0000147.

[20] E. Hennessy, J. T. Ornstein, C. D. Economos, J. B. Herzog, V. Lynskey, E. Coffield, R. A. Hammond, Designing an AgentBased Model for Childhood Obesity Interventions: A Case Study of ChildObesity180., Preventing chronic disease 13 (2016) E04. doi:10.5888/pcd13.150414

[21] B. Guinhouya, G. K. Apété, H. Hubert, The determinants of habitual physical activity (HPA) in children: update and implications for care and prevention options in pediatric overweight/obesity, Revue d'épidémiologie et de santé publique 58 (1) (2010) 49-58. doi:10.1016/j.respe.2009.10.007.

[22] R. A. Hammond, J. T. Ornstein, A model of social influence on body mass index., Annals of the New York Academy of Sciences 1331 (2014) 1-9. doi:10.1111/nyas.12344

[23] M. J. Widener, S. S. Metcalf, Y. Bar-Yam, Agent-based modeling of policies to improve urban food access for low-income populations, Applied Geography 40 (2013) 1-10. doi:10.1016/ j.apgeog.2013.01.003

[24] A. H. Auchincloss, R. L. Riolo, D. G. Brown, J. Cook, A. V. Diez Roux, An agent-based model of income inequalities in diet in the context of residential segregation, American Journal of Preventive Medicine 40 (3) (2011) 303-311. doi:10.1016/j. amepre.2010.10.033

[25] L. M. Garcia, A. V. Diez Roux, A. C. Martins, Y. Yang, A. A. Florindo, Development of a dynamic framework to explain population patterns of leisure-time physical activity through agent-based modeling, International Journal of Behavioral Nutrition and Physical Activity 14 (1) (2017) 1-8. doi:10.1186/ s12966-017-0553-4

[26] D. Zhang, P. J. Giabbanelli, O. A. Arah, F. J. Zimmerman, Impact of Different Policies on Unhealthy Dietary Behaviors in an Urban Adult Population: An Agent-Based Simulation Model, American Journal of Public Health 104 (7) (2014) 12171222. doi:10.2105/AJPH. 2014.301934

[27] Y. Yang, A. V. Diez-Roux, Using an agent-based model to simulate children's active travel to school., The international journal of behavioral nutrition and physical activity 10 (1) (2013) 67 . doi:10.1186/1479-5868-10-67

[28] Y. Yang, A. Diez-Roux, K. R. Evenson, N. Colabianchi, Examining the impact of the walking school bus with an agent-based model., American journal of public health 104 (7) (2014) 1196203. doi:10.2105/AJPH.2014.301896

[29] G. King, M. Law, S. King, P. Hurley, P. Rosenbaum, S. Hanna, M. Kertoy, N. Young, CAPE/PAC: Children's Assessment of Participation and Enjoyment \& Preferences for Activities of Children, NCS Pearson, Inc., 2004.

[30] J. B. Moore, Z. Yin, J. Hanes, J. Duda, B. Gutin, P. Barbeau, Measuring Enjoyment of Physical Activity in Children: Validation of the Physical Activity Enjoyment Scale, Journal of Applied Sport Psychology 21 (sup1) (2009) S116-S129. arXiv:NIHMS150003 doi:10.1080/10413200802593612

[31] H. Rogers, T. Morris, M. Moore, A qualitative study of the achievement goals of recreational exercise participants, The Qualitative Report 13 (4) (2008) 706-734.

[32] B. Engel-Yeger, A. Engel, A. Kessel, Differences in leisure activities between children with allergic rhinitis and healthy peers, International Journal of Pediatric Otorhinolaryngology 74 (12) (2010) 1415-1418. doi:10.1016/j.ijporl.2010.09.021

[33] J. Zhang, L. Tong, P. Lamberson, R. Durazo-Arvizu, A. Luke, D. Shoham, Leveraging social influence to address overweight and obesity using agent-based models: The role of adolescent social networks, Social Science \& Medicine 125 (2015) 203-213. doi:10.1016/j.socscimed.2014.05.049

[34] M. G. Orr, S. Galea, M. Riddle, G. A. Kaplan, Reducing racial disparities in obesity: Simulating the effects of improved education and social network influence on diet behavior, An- 
nals of Epidemiology 24 (8) (2014) 563-569. doi:10.1016/j.j05 annepidem.2014.05.012

[35] S. J. Chen, C. L. Hwang, Fuzzy Multiple Attribute Decision Making: Methods and Applications, Springer-Verlag New York, Inc., Secaucus, 1992.

[36] J. Fülöp, Introduction to decision making methods, BDEI-3910 Workshop, Washington (2005) 1-15.

[37] C. L. Hwang, K. Yoon, Multiple Attribute Decision Making, Vol. 186 of Lecture Notes in Economics and Mathematical Systems, Springer Berlin Heidelberg, Berlin, Heidelberg, 1981. doi:10.1007/978-3-642-48318-9

[38] L. Zedeh, Knowledge representation in fuzzy logic, IEEE Transactions on Knowledge and Data Engineering 1 (1) (1989) 89100. doi:10.1109/69.43406

[39] Z. Xu, Linguistic Aggregation Operators: An Overview, in: Fuzzy Sets and Their Extensions: Representation, Aggregation and Models, Vol. 220, Springer Berlin Heidelberg, Berlin, Hei口 delberg, 2008, pp. 163-181. doi:10.1007/978-3-540-73723-0_ 9

[40] F. Herrera, E. Herrera-Viedma, Aggregation operators for linguistic weighted information, IEEE Transactions on Systems, Man, and Cybernetics Part A:Systems and Humans. 27 (5) (1997) 646-656. doi:10.1109/3468.618263

[41] R. Yager, On ordered weighted averaging aggregation operators in multi criteria decision making, IEEE Trans. Syst. Man Cybern. 18 (1) (1988) 183-190. doi:10.1109/21.87068

[42] L. Zadeh, The concept of a linguistic variable and its application to approximate reasoning-II, Information Sciences 8 (4) (1975) 301-357. doi:10.1016/0020-0255(75)90046-8

[43] M. Delgado, J. L. Verdegay, M. A. Vila, On aggregation operations of linguistic labels, International Journal of Intelligent Systems 8 (1993) 351-370. doi:10.1002/int.4550080303

[44] F. Herrera, L. Martinez, A 2-tuple fuzzy linguistic representation model for computing with words, Fuzzy Systems, IEEE Transactions on 8 (6) (2000) 746-752. doi:10.1109/91.890332.

[45] R. Aziza, A. Borgi, H. Zgaya, B. Guinhouya, A Multi-agent Simulation: The Case of Physical Activity and Childhood Obesity, in: Distributed Computing and Artificial Intelligence (DCAI), 11th International Conference SE - 42, Vol. 290 of Advances in Intelligent Systems and Computing, Springer, Cham, 2014, pp.

875 359-367. doi:https://doi.org/10.1007/978-3-319-07593-8_ 42

[46] L. A. Garrison, D. S. Babcock, Alcohol consumption among college students: An agent-based computational simulation, Complexity 14 (6) (2009) 35-44. doi:10.1002/cplx.20259

[47] WHO, New Child Growth Standards, World Health Organizatior doi:10.1037/e569412006-008.

[48] M. Behzadian, S. Khanmohammadi Otaghsara, M. Yazdani, J. Ignatius, A state-of the-art survey of TOPSIS applications, Expert Systems with Applications 39 (17) (2012) 13051-13069. doi:10.1016/j.eswa.2012.05.056

[49] E. Cables, M. S. García-Cascales, M. T. Lamata, The LTOPSIS: An alternative to TOPSIS decision-making approach for linguistic variables, in: Expert Systems with Applications, Vol. 39, 2012, pp. 2119-2126. doi:10.1016/j.eswa.2011.07.119

[50] X.-H. Li, S. Lin, H. Guo, Y. Huang, L. Wu, Z. Zhang, J. Ma, H.-J. Wang, Effectiveness of a school-based physical activity intervention on obesity in school children: a nonrandomized

n controlled trial., BMC public health 14 (1) (2014) 1282. doi: 10.1186/1471-2458-14-1282

[51] N. a. Khan, L. B. Raine, E. S. Drollette, M. R. Scudder, M. B. Pontifex, D. M. Castelli, S. M. Donovan, E. M. Evans, C. H. Hillman, Impact of the FITKids physical activity intervention on adiposity in prepubertal children., Pediatrics 133 (4) (2014) e875-83. doi:10.1542/peds.2013-2246.

[52] Y.-P. Li, X.-Q. Hu, E. G. Schouten, A.-L. Liu, S.-M. Du, L.-Z. Li, Z.-H. Cui, D. Wang, F. J. Kok, F. B. Hu, G.-S. Ma, Report on Childhood Obesity in China (8): Effects and Sustainability of Physical Activity Intervention on Body Composition of Chinese Youth, Biomedical and Environmental Sciences 23 (3) (2010) 180-187. doi:10.1016/S0895-3988(10)60050-5
[53] L. R. Peralta, R. a. Jones, A. D. Okely, Promoting healthy lifestyles among adolescent boys: the Fitness Improvement and Lifestyle Awareness Program RCT., Preventive medicine 48 (6) (2009) 537-42. doi:10.1016/j.ypmed.2009.04.007.

[54] I. Anastasiadi, G. Tzetzis, Construct validation of the Greek version of the Children's Assessment of Participation and Enjoyment (CAPE) and Preferences for Activities of Children (PAC)., Journal of physical activity \& health 10 (4) (2013) 523-532.

[55] A. R. Ness, S. D. Leary, C. Mattocks, S. N. Blair, J. J. Reilly, J. Wells, S. Ingle, K. Tilling, G. D. Smith, C. Riddoch, Objectively measured physical activity and fat mass in a large cohort of children., PLoS medicine 4 (3) (2007) e97. doi:10.1371/journal.pmed.0040097 